\title{
Squamous cell carcinoma of lung associated with rare cutaneousparaneoplastic syndrome-systemic lupus erythromatosus (SLE)
}

\begin{abstract}
The burden of lung cancer is rising at alarming rates. Their coexistence with cutaneousparaneoplastic syndromes is not uncommon. We observed nests of malignant cells from the lung biopsy, hyperkeratosis with lymphocytic infiltration at the dermoepidermal junction from the biopsies of the skin lesion in an elderly man with history of addiction to nicotine and tobacco smoke. An immunological assay targeting auto-antibodies dsDNA and ANA were identified, possibly establishing an unknown and uncommon mechanism common to both lung cancer and Systemic Lupus Erythematosus (SLE).
\end{abstract}

\author{
Volume 7 Issue 2 - 2017 \\ Siddanna Rm,' Nihanthy Sreenath,' Shekar \\ 'Department of Radiation Oncology, Kidwai Memorial Institute \\ of Oncology, India \\ ${ }^{2}$ Department of Medical Oncology, HCG-Bangalore Institute of \\ Oncology, India \\ ${ }^{3}$ Departmentof Radio Diagnosis, Belgum Institute of Medical \\ sciences, India
}

Correspondence: Nihanthy Sreenath, Kidwai Memorial Institute of Oncology, Department of Radiation Oncology, India, Email nihanthyds@gmail.com

Received: August 09, 2017 | Published: August 23, 2017

\section{Article}

In the early $20^{\text {th }}$ century, lung cancer was considered to be very rare $^{1}$ but now it is the leading cause of cancer death worldwide, reaching an epidemic proportion. ${ }^{2}$ The report of GLOBOCAN series by the International Agency for Research on cancer summarizes the highest incidences and mortality for lung cancer throughout the globe with significant differences in the patterns and presentations from region to region. ${ }^{3}$

Various etiological factors have been identified, but the most striking causative factors are cigarette smoke, tobacco smoke and exposure to inhaled carcinogens. The ratios of smokers versus non smokers have been compared and are at a raise. There are variations in the demographic patterns of distributions in the histology's of lung cancers. In many Western countries adenocarcinoma has become the commonest ${ }^{4,5}$ while most of the Indian studies have described squamous cell carcinoma as the commonest histology in both men and women ${ }^{6,7}$ however change in the pattern has emerged. ${ }^{8,9}$

Their associations with various cutaneousparaneoplastic syndromes are not uncommon in European, American and Australian subcontinents but are extremely rare in Asian population especially in Indians. Tylosis, Dermatomyositis and polymyositis, Vasculitis, SLE, are few to name. ${ }^{10-13}$

SLE is an autoimmune, multisystem disease, rarely seen in lung cancers. In some of the reported cases it may precede diagnosis of malignancy while in few it may occur late in the course of disease or can be precipitated by exposure to immunosuppressive drugs or with history of active smoking. Compared with the general population, overall there is a slight surge (10-15\%) in the incidence of lung cancer in patients diagnosed with SLE. ${ }^{14}$

\section{Case report}

A71year old man, presented with history of excoriation and ulceration of skin over the dorsum of hands, feet, anterior and posterior surfaces of his chest (Figures 1A-1C), fatigue and dry cough of 10months duration. He was a chronic smoker with smoking index of greater than 40 pack years and was on beta blockers and ACE inhibitors for ischemic heart disease. Chest roengtogram showed alveolar infiltrates in the left upper zone. In suspicious of malignancy, FDG PET/CT scan was advised which showed a metabolically active $7.6 \times 5.6 \times 5 \mathrm{~cm}$ lesion in the posterior segment of the upper lobe of the left lung, with multiple bilaterally enlarged mediastinal lymph nodes, largest measuring $4.3 \mathrm{X} 2.3 \mathrm{~cm}$ seen in the subcarinal region (Figures 1D-1F). CT guided core biopsy from lung lesion showed moderately differentiated squamous cell carcinoma with extensive necrosis (Figure 2A) (Figure 2B). Immunohistochemistry staining of the neoplastic cells expressed p63 (Figure 2F) and were negative for TTF1. Biopsy from the skin lesion showed hyperkeratosis with follicular plugging and basal cell vacuolation with superadded dermatophyte infection (Figure 2C) (Figure 2D). Immunological studies were performed; positivity for anti-nuclear antibody and dsDNA were detected (Figure 2E).

Rarity in occurrence, this case posed a diagnostic perplexity. With series of investigations performed, the final diagnosis of carcinoma lung with coexistence of paraneoplastic cutaneous syndrome - Lupus erythematosus was established. Patient was planned on six to eight cycles of palliative chemotherapy, received only three cycles of Parenteral Nabpaclitaxel 100mg with parenteral Carboplatin 450mg once in 4weeks as the patient could not tolerate further cycles of chemotherapy due to drop in the WBC counts, chemotherapy was withheld in view of grade IV leucopenia. In due course of time, there was complete resolution of the cutaneous lesions (Figure 3A-3D). Patient was subjected for interim FDG PET imaging which showed partial response to treatment, as per RECIST criteria. ${ }^{15}$ In view of complete response of skin lesions and partial response of lung lesions, patient was planned for palliative radiotherapy. He received a biological equivalent dose of $60 \mathrm{~Gy}$ to the gross tumor volume on linac via IMRT technique. 

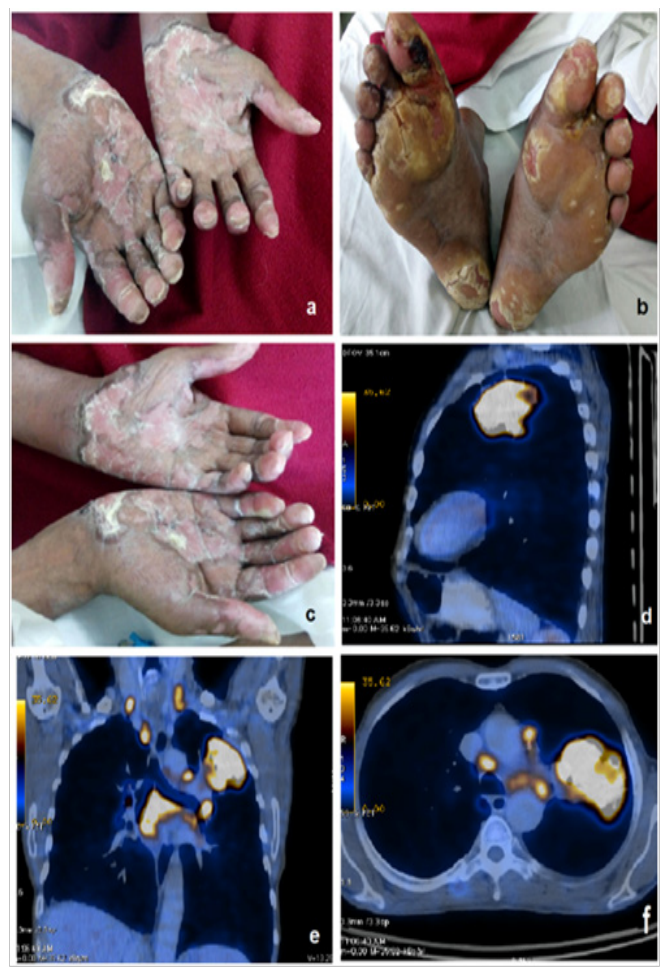

Figure I excoriation and ulceration of skin over the dorsum of hands, feet, anterior and posterior surfaces of his chest.
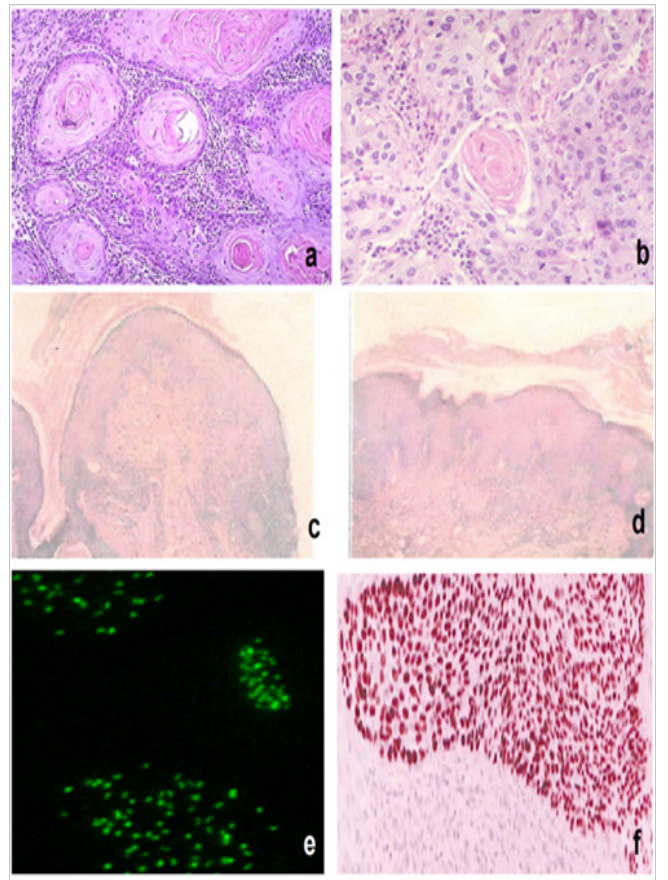

Figure 2 CT guided core biopsy from lung lesion showed moderately differentiated squamous cell carcinoma with extensive necrosis.

Patient improved considerably and was asymptomatic at the completion of treatment with complete resolution of skin lesions following chemotherapy. Repeat PET/CT imaging was done to assess the response of the lung lesion post radiotherapy. The scan revealed complete response of the lung lesion to treatment (Figure 3E) (Figure $3 \mathrm{~F})$.
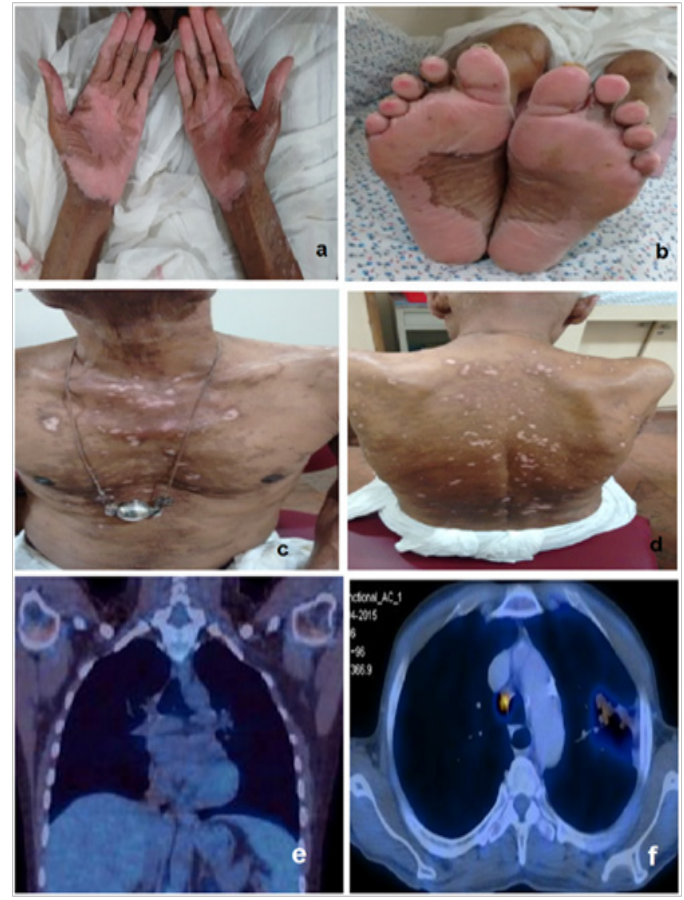

Figure 3 Chemo- radiotherapy.

\section{Discussion}

The cytogenetic studies of lung cancer have identified many chromosomal changes and structural aberrations which include deletions and translocations. Various mutations seen are either due to activation of the dominant cellular proto-oncogenes of the ras and myc family or the inactivation of the tumour suppressor genes or both. ${ }^{16,17}$

The genetics of SLE is T-cell DNA demethylation, which promotes a condensed chromatin configuration that is inaccessible to transcription factors. The methylation patterns are then replicated each time a cell divides by DNA methyltransferase 1. Inhibition of T-cell DNA methylation converts helper CD4+ T cells into autoreactive, cytotoxic and pro-inflammatory cells that cause lupuslike autoimmunity. ${ }^{18}$

The pathogenesis that associates SLE and lung cancer remains undetermined, various hypotheses have been established like the genetic susceptibility, predisposing to the development of both SLE and lung cancer and the development of the former may also be facilitated by smoking. ${ }^{19,20}$ Another theory associated is, through the development of fibrotic lung disease, suggesting that pneumonitis could lead to chronic inflammation and could induce DNA damage, leading to cytogenetic abnormalities that may lead to the development of lung cancer. ${ }^{21,22}$ Yet another theory states that, SLE and the drugs used in the treatment, might serve as a risk factor for malignancy by the cytotoxic effect exhibited by the drug. ${ }^{23}$ Lung cancers and their counter immune mechanism may lead to various autoantibody productions with initiation or precipitation of SLE, which can severely affect the organ function and quality of life, thus treating the underlying cancer may be the utmost priority for better outcomes and for survival benefits.

There are very few reported cases of SLE coexisting with lung cancer and the aetiology is by far unknown. A multicentre international cohort study was performed by Bin $\mathrm{J}$ et al., ${ }^{24}$ to provide a brief report 
of the lung cancer cases from an SLE cohort and concluded that histological pattern of distribution of lung cancer were similar in SLE cohort and in general population but incidence of malignancy was slightly high in SLE cohorts when compared with general population with a standardized incidence rate 1.37, but hasn't thrown light on the exact mechanism of coexistence. ${ }^{24}$ Incidence of SLE and lung cancer was higher in women. ${ }^{25-28}$ The reported incidence of lung cancers coexisting with SLE or vice verse in Indian population compared to western population are very few, the incidence is being less than $2 \%{ }^{1}$

In our report, with the aid of supplementary investigations we could arrive at a final diagnosis but the treatment options were considered with a grain of salt due to the immuno-compromised status of the patient.

With utmost care and precaution Chemotherapy was initiated and fortunately Chemotherapy neither precipitated nor flared his cutaneous symptoms and to our surprise the immune-compromised patient responded very well for Chemotherapy and his skin lesions resolved completely but drop in his WBC counts, raised a question for further continuation and thus Chemotherapy was withheld. Radiotherapy was supplemented to treat his partially responded lung lesions; with a slight dilemma in mind regarding the interaction of radiation with the skin lesions. High precision and daily monitoring, Radiotherapy was delivered successfully. Radiation nor aggravated or precipitated the skin lesions and with complete response of lung lesions.

However, we were unable to conclusively establish the sequence of appearance of the co-existent conditions, the precipitating factors involved or whether the malignant transformation was due SLE or malignancy induced immune alteration precipitated SLE?

This is a rare case of lung cancer with co-existing immunecompromised condition-SLE, which resolved with chemoradiotherapy (Figure 3).

\section{Acknowledgements}

None.

\section{Conflict of interest}

The author declares no conflict of interest.

\section{References}

1. Nath V, Grewal KS. Cancer in India. Indian journal Medical research. 1935;23(1):149-190.

2. Khuri FR, Herbst RS, Fossells FV. Emerging therapies in non-small cell lung cancer. Ann Oncol. 2001;12(6):739-744.

3. Ferlay J, Shin HR, Bray F, et al. Estimates of worldwide burden of cancer in 2008: GLOBOCAN 2008. Int J Cancer. 2010;127(12):2893-2917.

4. Noronha V, Dikshit R, Raut N, et al. Epidemiology of lung cancer in India: focus on the differences between non-smokers and smokers; a single-centre experience. Indian J Cancer. 2012;49(1):74-81.

5. Malik PS, Sharma MC, Mohanti BK, et al. Clinico-pathological profile of lung cancer at AIIMS: A changing paradigm in India. Asian Pac J Cancer Prev. 2013;14(1):489-494.

6. Valaitis J, Warren S, Gamble D. Increasing incidence of adenocarcinoma of the lung. J Cancer. 1981;47(5):1042-1046.

7. Janssen-Heijnen MLG, Coebergh J-WW. Epidemiology of lung cancer in Europe. J Lung Cancer. 2003;41(3):245-258.
8. Behera D, Balamugesh T. Lung cancer in India. Indian J Chest Disease and Allied Scienes. 2004;46(4):269-281.

9. Singh N, Aggarwal AN, Gupta D, et al. Unchanging clinico-epidemiological profile of lung cancer in north India over three decades. $J$ Cancer Epidemiol. 2010;34(1):101-104.

10. Stefanie Heinemann, Peter Zabel, Hans-Peter Hauber. Paraneoplastic syndromes in lung cancer. Medical Clinic. Research Center Borstel. 2008;8:687-698.

11. Hansen M. Paraneoplastic syndromes and tumor markers for small cell and non small cell lung cancer. J CurrOpin Oncol. 1990;2(2):345-351.

12. Buchbinder R, Hill CL. Malignancy in patients with inflammatory myopathy. J Current rheumatol rep. 2002;4(5):415-426.

13. Gabrilovich M, Raza M, Dolan S, et al. Paraneoplastic polymyos it is associated with squamous cell carcinoma of the lung. J Chest. 2006;129(6):1721-1723.

14. Bernatsky S, Ramsey-Goldman R, Clarke AE. Revisiting the issue of malignancy risk in systemic lupus erythematosus. J Curr Rheumatol Rep. 2005;7(6):476-481.

15. Eisenhauera EA, Therasse p, Bogaerts J, et al. New response evaluation criteria in solid tumours- revised RECIST guideline (version 1.1). EurJ cancer. 2009;45(2):228-247.

16. Jocket KH, Ahrens W, Wichmann HE, et al. Occupational and environmental hazards associated with lung cancer. Int J Epidemiol. 1992;21(2):202-213.

17. Minna JD. Genetic events in the pathogenesis of lung cancer. $J$ Chest. 1989;96(1 Suppl):17S-23S.

18. Javierree BM, Richardson B. A new epigenetics challenge: systemiclupuserythomatosus. Adv Exp Med Biol. 2011;711:117-136.

19. Archontogeorgis K, Bouros Department of Pneumonology, Medical School, Democritus University of Thrace, 68100 Alexandroupolis, Greece.

20. Laboratory of Hygiene and Environmental Protection, Medical School, Democritus University of Thrace, 68100 Alexandroupolis, Greece.

21. Kiyohara C, Washio M, Horiuchi T, et al. Cigarette smoking: N-acetyltransferase 2 polymorphisms and systemic lupus erythematosus in a Japanese population. J Lupus. 2009;18(7):630-638.

22. Hubbard R, Venn A, Lewis S, et al. Lung cancer and cryptogenic fibrosingalveolitis. A population based cohort study. Am J of Respir Crit Care Med. 2000;161(1):5-8.

23. Bernatsky S, Joseph L, Boivin JF, et al. The relationship between cancer and medication exposures in systemic lupus erythaematosus:a case-cohort study. Ann Rheum Dis. 2008;67(1):74-79.

24. Bin J. Lung cancer in SLE. International journal for Lung cancer and other thoracic malignancies. 2007;56:303-306.

25. Bernatsky S, Boivin JF, Joseph L, et al. An international cohort study of cancer in systemic lupus erythematosus. J Arthritis and Rheumatism. 2005;52(5):1481-1490.

26. Ragnarsson O, Grondal G, Steinsson K. Risk of malignancy in an unselected cohort of Icelandic patients with systemic lupus erythematosus. JLupus. 2003;12(9):687-691.

27. Ramsey-Goldman R, Mattai SA, Schilling E, et al. Increased risk of malignancy in patients with systemic lupuserythematosus. J Investig Med. 1998;46(5):217-222.

28. Mellemkjaer L, Andersen V, Linet MS, et al. Non-Hodgkin's lymphoma and other cancersamong a cohort of patients with systemic lupus erythematosus. J Arthritis Rheum. 1997;40(4):761-768. 